\title{
Use of Helical Transport Channels for Bunch Recombination
}

\author{
David Neuffer ${ }^{\#}$, Katsuya Yonehara ${ }^{\#}$ and Cary Yoshikawa* \\ ${ }^{\#}$ Fermilab \\ PO Box 500, Batavia IL 60510 \\ "Muons, Inc. \\ Batavia IL 60510
}

March 2010

\begin{abstract}
Cooling scenarios for a high-luminosity Muon Collider require bunch recombination for optimal luminosity. In this report we note that the tunable chronicity property of a helical transport channel (HTC) makes it a desirable component of a bunch recombiner. A large chronicity HTC is desirable for the bunch recombining transport, while more isochronous transport may be preferred for rf manipulations. Scenarios for bunch recombination are presented, with initial 1-D simulations, in order to set the stage for future 3-D simulation and optimization. HTC transports may enable a very compact bunch recombiner.
\end{abstract}

\section{Introduction}

For a $\mu^{+}-\mu^{-}$collider, muons from a high intensity production system must be cooled into short, intense bunches for maximal luminosity.[1, 2] The muons are produced from bunches of protons that are focused onto a target to produce pions, that then decay into muons. The muons are produced within a very large phase space that must be compressed and cooled to obtain high-luminosity parameters. Ref. [3] presents a scenario for this capture and cooling process. Longitudinally, the muons are initially captured into a string of rf bunches, and kept within those bunches until cooled to much reduced emittances, both longitudinally and transversely. At that point is desirable to recombine the bunches, recapturing in a single bunch with large longitudinal acceptance. That single bunch would then receive further cooling toward collider densities.

Fig. 1 shows an overview of the muon collider system and figure 2 shows the progression of transverse and longitudinal emittances through the muon cooling system. As displayed in those figures and detailed in ref [2], the muons are produced with initial emittances of $\sim 0.02 \mathrm{~m}$ transverse and $\sim 0.4 \mathrm{~m}$ longitudinally ( 0.03/bunch) and are captured in $\sim 12$ bunches (200MHz). The bunches are cooled to $\sim 0.0015 \mathrm{~m}$ transverse and $\sim 0.002 /$ bunch longitudinally, where they are then merged into a single bunch. (step 5 of fig. 2). The merged bunch is further 
cooled and emittance exchanged to $\sim 0.07 \mathrm{~m}$ longitudinal and 0.00003 transverse emittances, suitable for high-luminosity collisions.

The merger into a single bunch was imagined to require a very long rf and transport section, with losses of $\sim 50 \%$ from $\mu$ decay alone. In this report we note that the large chronicity that is possible in a helical transport channel enables performing this bunch combination in a much more compact system that should be more efficient.

\section{Quasiisochronous and Anisochronous HTC for Bunch Recombination}

In ref. [4], Derbenev and Johnson proposed the use of helical cooling channels for cooling of muon beams to small emittances. In this section we note that the tunable chronicity of helical channels can also be used for bunch combination and consider the use of isochronous and anisochronous helical transport channels for the bunch recombination section of a muon cooling channel.

In a possible muon collection and cooling scheme,[3] the muons are first captured in a string of $\sim 200 \mathrm{MHz}$ bunches ( $\sim 12$ bunches)[5] and then cooled in a sequence of 6D cooling channels to a much reduced transverse emittance and a much reduced longitudinal emittance per bunch. At the end of such a channel the transverse emittance would be $\sim 0.001 \mathrm{~m}$ (normalized, rms), and the beam would be in longitudinal emittances of $\sim 0.002 \mathrm{~m}$ per bunch.

We propose using a helical transport channel (HTC) in order to recombine these bunches, and we label this system a Bunch Recombiner (BR). The initial section of the system would be an isochronous system in which rf (or induction acceleration) would be used to give the bunches different energies, placing the head bunches at a higher energy than the trailing bunches. This is then followed by an anisochronous drift that moves the bunches together, forming a single bunch with an enlarged energy spread that is captured within a single rf bucket. This drift is designed such that larger energy particles have a much larger path length than lower energy particles, so that the bunches combine in a relatively short length. Because of its broadly tunable chronicity, an HTC can be readily designed with this property. That enlarged energy spread bunch would then be cooled by the downstream cooling as part of a system that would reduce the beam emittances to collider values.

As an illustration of the proposed method, we consider the following example, which is also displayed in fig. 4. We start with a train of twelve bunches, each with longitudinal rms emittance of $\sim 0.0025$, spaced at $200 \mathrm{MHz}$. (c $\tau=1.5 \mathrm{~m}$ ) An rf system within a quasisochronous HTC moves the first bunch kinetic energy to $250 \mathrm{MeV}$, with each following bunch $10 \mathrm{MeV}$ lower in energy, so that bunch 12 has a central kinetic energy of $\sim 140 \mathrm{MeV}$. At this point the bunches have an energy width of $\sigma_{E}=2.5 \mathrm{MeV}$ and $\sigma_{z}=10 \mathrm{~cm}$. The bunches then drift through an anisochronous channel to a short single bunch that fits within a $\sim 200 \mathrm{MHz}$ rf bucket. For this transport, we choose an HTC of the type presented in ref. [4], which has a period of $\lambda=1.6 \mathrm{~m}$, the parameter $\kappa=k a=1$, with a solenoidal field of $B=4.2 \mathrm{~T}$, a dipole field $\mathrm{b}_{\mathrm{d}}=0.75$, and $b_{q}=-0.45$. At these parameters the anisochronous parameter $\eta$ is:

$$
\eta=\frac{\sqrt{1+\kappa^{2}}}{\gamma \beta^{3}}\left[\frac{\hat{D} \kappa^{2}}{1+\kappa^{2}}-\frac{1}{\gamma^{2}}\right] \cong 0.43
$$

Note that $\hat{D}$ is $\sim 1.7$, and the dispersion ( $\hat{D}$ a) is $\sim 0.44 \mathrm{~m}$, in this example. The (linearized) equation of motion is: 


$$
\delta(c \tau)=\eta \frac{\delta E}{m_{\mu} c^{2}} z,
$$

where $z$ is distance along the HTC.

At these parameters an HTC of $\sim 37 \mathrm{~m}$ is sufficient to recombine the 12 bunches. This is a dramatic improvement over the bunch recombiner strategy presented in ref. [3], where a 340m transport is required for bunch recombination.[6] At that point a modest rf system can be introduced to hold the beam within a bunch, within a smaller chronicity transport. (A wedge absorber could also be introduced to reduce the energy spread, at a cost of increased transverse emittance.) Downstream cooling systems will then cool that bunch to the smaller emittances needed for a collider.

This initial example was developed from parameters readily available in current HTC designs, where the designs are motivated by 3-D cooling considerations. We have not made a systematic attempt at reoptimizing the transport for bunch recombination. The R\&D included in phase 2 of this research would provide a more complete optimization of the system parameters.

\section{More Complete Scenario: Bunch Preparation}

The bunch recombination requires forming the cooled beam bunches into a string with different energies, with each bunch in a compact energy spread and moderate bunch length $(\sim 2.5 \mathrm{MeV} \times 10 \mathrm{~cm})$, with a $\sim 10 \mathrm{MeV}$ spacing between $200 \mathrm{MHz}$ bunches. In ref. 3 , this was to be done by a $5 \mathrm{MHz}$ rf system. This low frequency system would be limited in gradient and very expensive.

In this section we present a different approach to this problem. We would instead use an rf system with a frequency near $200 \mathrm{MHz}$. The difference between that frequency and the baseline would be used to place the centers of the bunches at different phases such that head bunches are accelerated and tail bunches are decelerated. (Changing the signs of the rf voltage and the chronicity allows one to use a frequency either above or below the baseline.) One expected that the initial bunches would have to be very short to avoid developing a large energy spread in the high frequency rf. However we found a solution where the high frequency rf naturally bunches the beam into the long-bunch small- $\delta \mathrm{E}$ format needed for the Bunch Recombiner drift.

We present the parameters of an initial reference example to demonstrate the method. The bunches from the upstream cooling channel enter the BR with $\sigma_{\mathrm{z}}=0.6 \mathrm{~cm}, \sigma_{\mathrm{E}}=33 \mathrm{MeV}$, with a mean kinetic energy of $200 \mathrm{MeV}$. (The cooler would naturally produce bunches with $\sigma_{\mathrm{z}}=1 \mathrm{~cm}, \sigma_{\mathrm{E}}=20 \mathrm{MeV}$, so we assume some bunch precompression.)

For the BR buncher we choose an rf wave length of ( $\lambda=1.5-0.03)$ meters, obtaining an rf wavelength of $\sim 204.08 \mathrm{MHz}$. The rf system would be $9.6 \mathrm{~m}$ long, with $\mathrm{V}_{\mathrm{rf}}=15 \mathrm{MV} / \mathrm{m}$ gradient and a transport chronicity of $\eta=0.05$. This transport could be a quasiisochronous HTC similar to those presented by C. Ankenbrandt et al.[7] At these parameters, we find the bunches become remarkably similar to those described in the previous section. $\left(\sigma_{\mathrm{z}}=\sim 10 \mathrm{~cm}, \sigma_{\mathrm{E}}=\right.$ 
$\sim 2.5 \mathrm{MeV}$, with $\sim 10 \mathrm{MeV}$ spacing.) This rf buncher is followed by a $33 \mathrm{~m}$ drift with $\eta=0.43$, just as described in the previous section.

This solution was initially somewhat fortuitous, but can probably be improved with some optimization. The function of the RB buncher is individual bunch acceleration coupled with phase-energy rotation of short $\delta z$, large $\delta \mathrm{E}$ bunches to large $\delta z$, small $\delta \mathrm{E}$ bunches $(\sim 1 / 4$ synchrotron oscillation) within the rf buckets. The simultaneous achievement of both goals is enabled by using HTC transports with chronicities $\eta$ matched to obtain the desired dynamics. We have not yet explored the effects of nonlinearities, such as amplitude-energy correlations, and nonlinear $\eta$, but we believe many such effects can be corrected with HTC design variations (quadrupole and sextupole components, etc.).

The total length of the system is $42 \mathrm{~m}$ in $\mathrm{z}$ (helical longitudinal length), and the rf gradient is a manageable $15 \mathrm{MV} / \mathrm{m}$ with $9.6 \mathrm{~m}$ length. This is dramatically less than previously considered.

This initial evaluation has only explored the 1-D beam dynamics at plausible parameters, within somewhat linearized systems. We will need further studies that include 3-D designs of HTC and rf transports, and 3-D calculations of the beam dynamics, with a more complete consideration of nonlinearities.

This initial example displays use of a quasiresonant off-energy phase rotation and bunching within a fixed frequency system, which is relatively constrained in its parameter choices. A more adiabatic solution using varying frequency rf (similar to that used in the baseline neutrino factory front end capture and phase-energy rotation[8]) is also possible. This more adiabatic variation could use lower rf gradients but would be longer and perhaps less efficient, and has not been worked out in detail.

\section{Acknowledgments}

We thank R. Johnson, C. Ankenbrandt and S. Geer for important contributions and discussions. Research supported by Department of Energy under contract no. DEAC0207CH11359 and SBIR Phase I funding DE-SC-0002739.

\section{REFERENCES}

1. The $\mu^{-}-\mu^{+}$Collaboration, Report BNL-52503, Fermi Lab-Conf. 96/092, LBNL-38946, presented at Snowmass96, (1996).

2. R. Palmer, “Muon Collider Progress”, NFMCC Tech. Note 529, presented at the 2009 Particle Accelerator Conference, Vancouver BC (2008).

3. R. Palmer et al., Proc. NuFACT08, $10^{\text {th }}$ Int. Conf. on Neutrino Factories, Super Beams, and Beta Beams, Valencia, Spain, July 2008.

4. Y. Derbenev and R. P. Johnson, Phys. Rev. STAB 8, 041002 (2005).

5. D. Neuffer and C. Yoshikawa, to appear in Proc. PAC09 Vancouver, B. C., May 2009.

6. R. Fernow, "Estimate of Front-End Magnetic Requirements, NFMCC Tech. Note 529 (2008).

7. C. Ankenbrandt, M. A. C. Cummings, R.P. Johnson, and C. Yoshikawa, D. Neuffer and K. Yonehara, "QuasiIsochronous Muon Capture”, to appear in Proc. PAC09 Vancouver, B. C., May 2009.

8. J. S. Berg, S. A. Bogasz, S. Caspi, J. Cobb, R. C. Fernow, J. C. Gallardo, S. Kahn, H. Kirk, D. Neuffer, R. Palmer, K. Paul, H. Witte, M. Zisman, "Cost-effective Design for a Neutrino Factory", Phys. Rev. STAB 9,011001(2006). 
Figure 1. An overview of the muon collider system, showing the muon production, phaseenergy rotation and cooling of 12 muon bunches, bunch recombination to 1 bunch, followed by more cooling and acceleration into a storage ring. (from ref. [2])

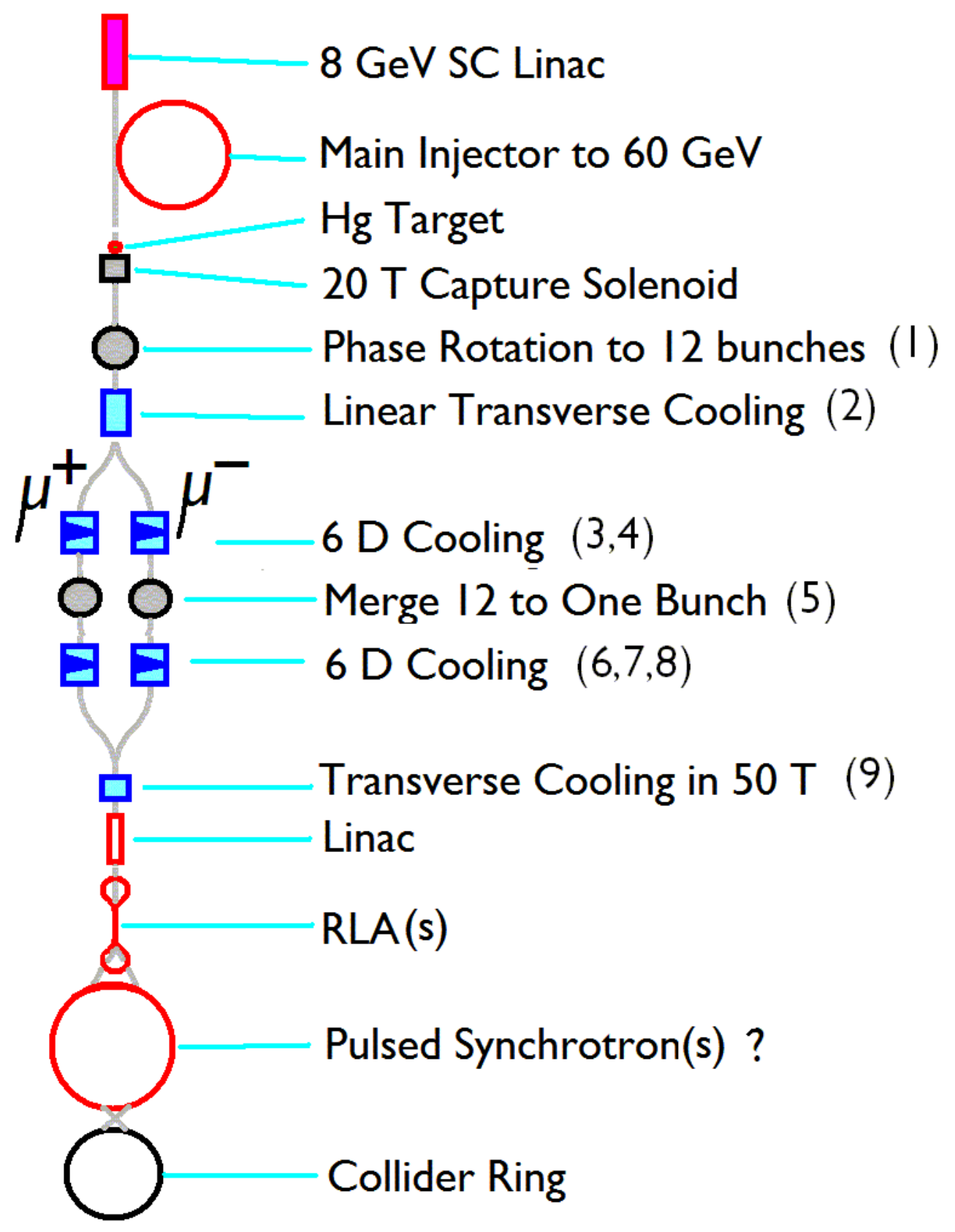


Figure 2. Progression of transverse and longitudinal emittances through a cooling system for a muon collider. The initial emittances of $\sim 0.02 \mathrm{~m}$ transverse and $0.4 \mathrm{~m}$ longitudinally ( $\sim 0.03 /$ bunch) are captured in $\sim 12$ bunches, cooled to $\sim 0.001 \mathrm{~m}$ transverse and $\sim 0.002 \mathrm{~m} / \mathrm{bunch}$ longitudinally. The merger of muon bunches to a single bunch is shown as step 5 . The merged bunch is further cooled to $\sim 0.001 \mathrm{~m}$ longitudinal and 0.0004 transverse, and then cooled and emittance exchanged to $\sim 0.07 \mathrm{~m}$ longitudinal and 0.00003 transverse emittances. (from ref. [2])

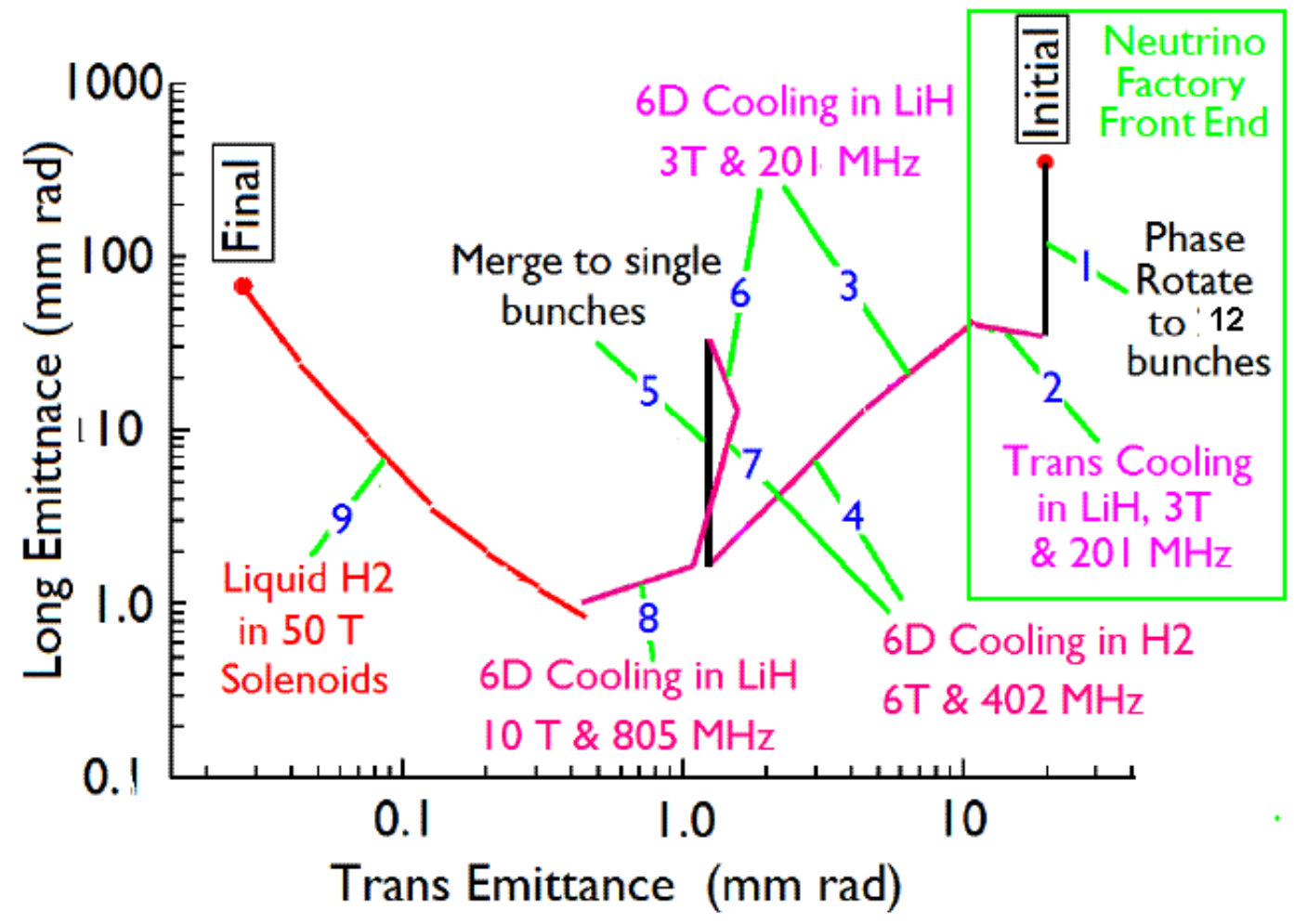


Figure 3: Schematic view of the HTC Bunch Recombiner:

A. Initially the beam is in a string of bunches.

B. In an isochronous channel, rf increases the energies of the head bunches relative to the tail bunches.

C. Drift in an anisochronous transport channel moves the bunches together, forming a single bunch with a large energy spread. A lower frequency rf system captures the bunches into a single bunch.
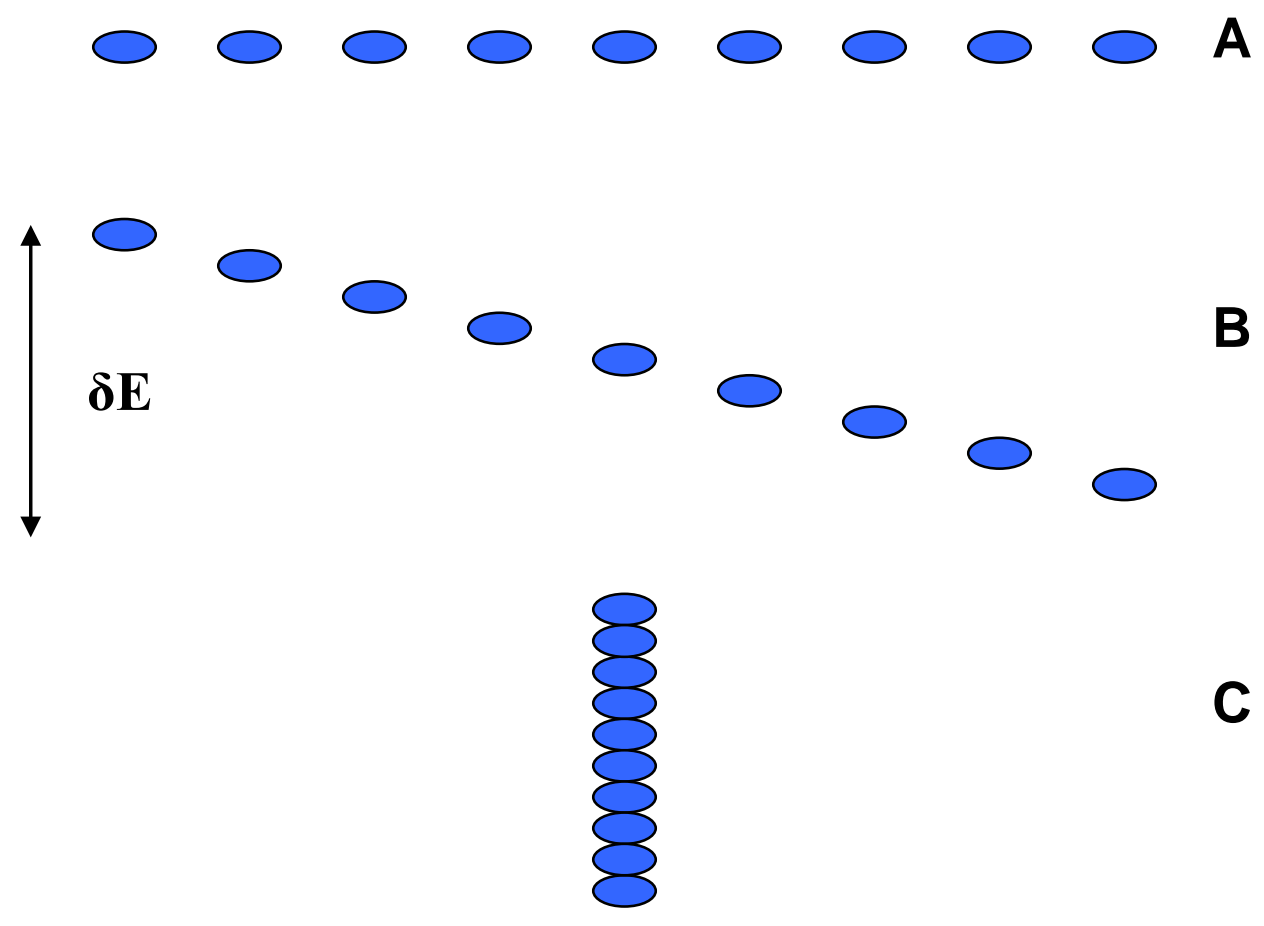
Figure 4: Results of a 1-D simulation of bunch recombination. In Figure A, a differential acceleration system has accelerated the head bunches with respect to the tail, with a separation of $10 \mathrm{MeV}$ per bunch. In Figure B, a $37 \mathrm{~m}$ HTC with $\eta=0.43$ has recombined the bunches. Figure $\mathrm{C}$ shows the beam as rebunched at $200 \mathrm{MHz}$ with a $10 \mathrm{MV} / \mathrm{m}$ buncher, showing $95 \%$ capture.

In each graph the vertical scale is kinetic energy ( 0 to $300 \mathrm{MeV})$ and the horizontal scale is c $\tau$ (-4 to $20 \mathrm{~m})$.

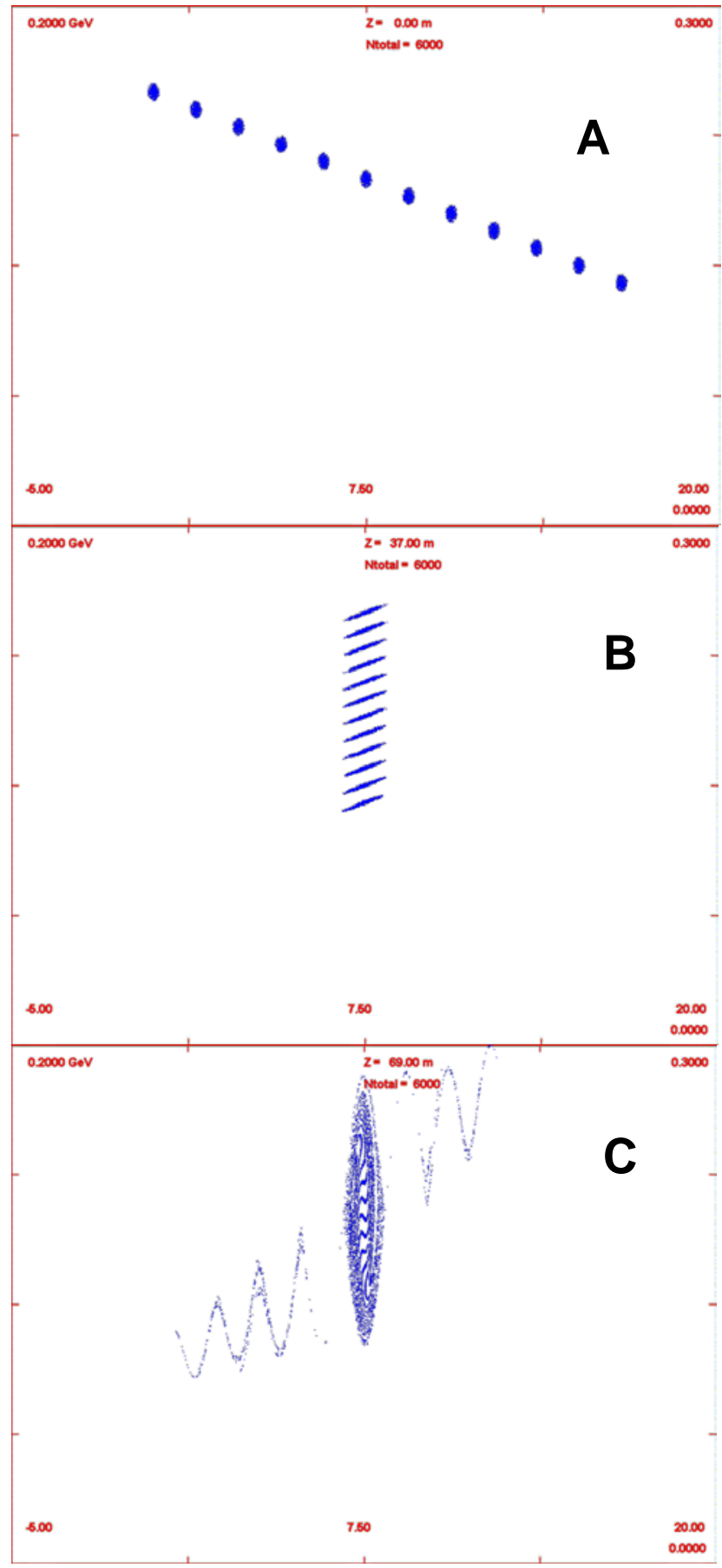


Figure 5. Overview of bunch recombiner with initial quasiisochronous bunch formation.

Figure $5 \mathrm{~A}$ shows a string of $200 \mathrm{MHz}$ rf bunches with $\varepsilon_{\mathrm{L}}=0.002 \mathrm{~m} / \mathrm{bunch}$.

Figure 5B shows bunches after $9.6 \mathrm{~m}$ of $204.08 \mathrm{MHz}$ rf within a quasiisochronous transport. $\eta=0.05, \mathrm{~V}^{\prime}=15 \mathrm{MV} / \mathrm{m}$.

Figure $5 \mathrm{C}$ shows the same beam after $32.5 \mathrm{~m}$ of drift at $\eta=0.43$.

Figure 5D shows the same beam after synchrotron oscillations within a $200 \mathrm{MHz}$ rf bucket. $\sim 95 \%$ of the initial beam is captured within that bucket.

In each graph the vertical scale is kinetic energy ( 0 to $300 \mathrm{MeV}$ ) and the horizontal scale is c $\tau$ (-4 to $20 \mathrm{~m})$.

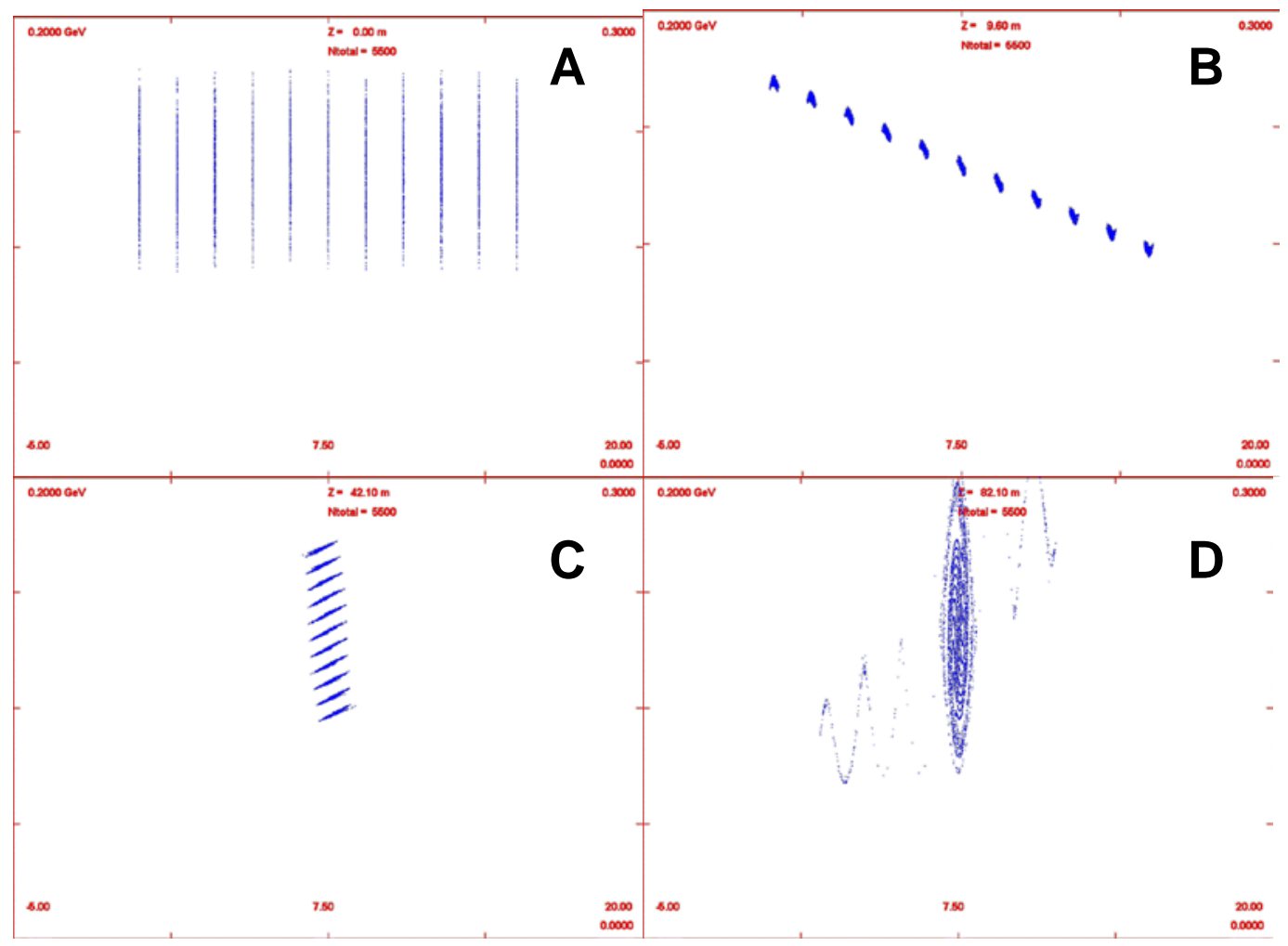

